ARTICLE

\title{
Exosome engineering for efficient intracellular delivery of soluble proteins using optically reversible protein-protein interaction module
}

\author{
Nambin Yim', Seung-Wook Ryu ${ }^{2}$, Kyungsun Choi ${ }^{1}$, Kwang Ryeol Lee ${ }^{2}$, Seunghee Lee ${ }^{1}$, Hojun Choi ${ }^{1}$, Jeongjin Kim³
} Mohammed R. Shaker ${ }^{4}$, Woong Sun ${ }^{4}$, Ji-Ho Park', Daesoo Kim³ ${ }^{3}$, Won Do Heo ${ }^{3,5,6}$ \& Chulhee Choi ${ }^{1,2,5}$

Nanoparticle-mediated delivery of functional macromolecules is a promising method for treating a variety of human diseases. Among nanoparticles, cell-derived exosomes have recently been highlighted as a new therapeutic strategy for the in vivo delivery of nucleotides and chemical drugs. Here we describe a new tool for intracellular delivery of target proteins, named 'exosomes for protein loading via optically reversible protein-protein interactions' (EXPLORs). By integrating a reversible protein-protein interaction module controlled by blue light with the endogenous process of exosome biogenesis, we are able to successfully load cargo proteins into newly generated exosomes. Treatment with protein-loaded EXPLORs is shown to significantly increase intracellular levels of cargo proteins and their function in recipient cells in vitro and in vivo. These results clearly indicate the potential of EXPLORs as a mechanism for the efficient intracellular transfer of protein-based therapeutics into recipient cells and tissues.

\footnotetext{
${ }^{1}$ Department of Bio and Brain Engineering, KAIST, Daejeon 34141, Korea. ${ }^{2}$ Cellex Life Sciences Inc., Daejeon 34141, Korea. ${ }^{3}$ Department of Biological Sciences, KAIST, Daejeon 34141, Korea. ${ }^{4}$ Department of Anatomy, Brain Korea 21 Program, Korea University College of Medicine, Seoul 02841, Korea. ${ }^{5}$ Cancer Metastasis Control Center, KAIST Institute for the Biocentury, KAIST, Daejeon 34141, Korea. ${ }^{6}$ Center for Cognition and Sociality, Institute for Basic Science (IBS), Daejeon 34047, Korea. Correspondence and requests for materials should be addressed to K.C. (email: ccbioks@kaist.ac.kr) or to C.C. (email: cchoi@kaist.ac.kr).
} 
$\mathrm{D}$ espite the long list of therapeutic proteins available for treating various human diseases, the vast majority of clinically available protein-based drugs, such as cytokines, hormones and monoclonal antibodies, have been limited to extracellular mechanisms of action. Intracellular proteins have been identified for their potential as biopharmaceutical drugs; however, many of the challenges associated with intracellular protein delivery have yet to be solved ${ }^{1}$. Protein transduction methods have been proposed to deliver recombinant proteins into target cells in vitro and in vivo ${ }^{2-4}$. Although the techniques are promising, exposure of the protein surface in solution and the low refolding rate of recombinant proteins in target cells remain important obstacles for the intracellular delivery of therapeutic proteins $^{5}$. Several lipid nanoparticle-mediated protein delivery methods have been postulated, as has protein encapsulation for protecting the proteins $s^{6,7}$. However, the absence of a separation mechanism between cargo proteins and lipid nanoparticles not only limits the efficiency of cytosolic delivery, preparation of these particles often involves complicated protein purification steps.

To address these limitations, we developed an optogenetically engineered exosome system EXPLORs (exosomes for protein loading via optically reversible protein-protein interactions) that can deliver soluble proteins into the cytosol via controlled, reversible protein-protein interactions (PPIs). Exosomes are natural cell-derived extracellular vesicles that originate from internal endocytic compartments and multi-vesicular bodies and participate in intercellular communication ${ }^{8}$. Recent studies have sought to use exosomes as a new method for the in vivo delivery of siRNA or miRNA to specific target tissues by systemic injection ${ }^{9-11}$. These methods were based on the passive loading of siRNAs or miRNAs into isolated exosomes by electrophoresis, a method poorly suited for loading of macromolecular proteins. Here we propose a novel protein-loading method in which cargo proteins can be actively loaded into exosomes through endogenous biogenesis processes, allowing for efficient delivery into the cytosol of target cells through controllable, reversible detachment from the exosomes.

To achieve the controllable, reversible loading and delivery of target proteins into the exosome, we selected a photoreceptor cryptochrome 2 (CRY2), and CRY-interacting basic-helix-loophelix 1 (CIB1) protein module, originally identified in Arabidopsis thaliana, which regulates floral initiation via blue light-dependent phosphorylation ${ }^{12-14}$. We induced a transient docking of CRY2-conjugated cargo proteins to the exosomes by introducing CIBN (a truncated version of CIB1) ${ }^{14}$ conjugated with an exosome-associated tetraspanin protein $\mathrm{CD} 9$ and blue light illumination (Fig. 1a,b). Once the cargo proteins are introduced into the exosomes via the process of endogenous biogenesis, they can be detached from CD9-conjugated CIBN by removal of the illumination source, resulting in their release into the intraluminal space of the exosomes and enabling efficient delivery to the cytosolic compartment of target cells.

\section{Results}

Light-inducible PPI module for producing EXPLORs. We generated HEK293T cells that can produce EXPLORs loaded with mCherry (mCherry:EXPLOR) as a target protein by introducing vectors for two fusion proteins: CIBN-conjugated enhanced green fluorescent protein (EGFP)-tagged CD9 (CIBN-EGFP-CD9) and mCherry-tagged CRY2 (mCherry-CRY2). A single pulse irradiation of a 488-nm laser induced rapid movement of mCherry-CRY2 from the cytosolic compartment to the plasma membrane and intracellular compartments where the CIBN-EGFP-CD9 proteins were co-localized (Fig. 1c and Supplementary Fig. 1a). Similar results were obtained by the introduction of another photoreceptor module GIGANTEA, and
LOV (light-, oxygen- and voltage-sensitive domain) proteins ${ }^{15}$ and other exosome-enriched tetraspanin proteins, such as CD63, CD81 and CD82 (Supplementary Fig. 1b,c). We could also observe light-induced co-localization of mCherry-CRY2 and CIBN-EGFP-CD9 by a cryo-immunogold electron microscopy analysis (Supplementary Fig. 2). Introduction of a mutant CRY2, mutated at an amino acid important in the photolyase function $(\mathrm{D} 387 \mathrm{~A})^{16}$, abrogated such light-induced co-localization of CIBN-EGFP-CD9 and mCherry-CRY2 (Supplementary Fig. 3). On termination of light stimulation, the association between CIBN-EGFP-CD9 and mCherry-CRY2 dissociated gradually within $10 \mathrm{~min}$, as described previously ${ }^{14}$ (Fig. 1d,e).

Light-dependent loading of proteins in EXPLORs. For production of mCherry:EXPLORs, we exposed transiently transfected HEK293T cells with continuous blue light illumination by installing a 460-nm light-emitting diode (LED; maximum power; $380 \mu \mathrm{W} \mathrm{cm}^{-2}$ ) in a $\mathrm{CO}_{2}$ incubator. After 48-h incubation with blue light illumination, EXPLORs were isolated from the supernatants by several different methods and confirmed for the hydrodynamic diameter by dynamic light scattering (Supplementary Fig. 4 and Supplementary Table 1). Immunoblotting results demonstrated that EXPLORs derived from optogenetically engineered HEK293T cells grown under blue light illumination contained significantly higher amounts of mCherryCRY2 protein $(84 \mathrm{kDa})$ than those obtained from cells grown in the dark (Fig. 2a and Supplementary Fig. 5). In particular, the highest efficiency in protein loading was seen in the power range from 20 to $50 \mu \mathrm{W} \mathrm{cm}{ }^{-2}$ (Fig. 2b). The threshold power of the light-induced PPI was measured at $\sim 15 \mu \mathrm{W} \mathrm{cm}{ }^{-2}$ by quantified laser stimulation (Supplementary Fig. 6) and at $\sim 20 \mu \mathrm{W} \mathrm{cm}{ }^{-2}$ by the LED light (Supplementary Fig. 7). Continuous irradiation with blue light for up to $48 \mathrm{~h}$ did not induce significant cell death (Supplementary Fig. 8), and we further confirmed that EXPLORs did not contain detectable DNA fragments typical of apoptotic bodies (Supplementary Fig. 9a). Because genetically modified cells may release exosomes packed with not only the encoded protein but also genetic material (pDNA and mRNA) encoding this protein ${ }^{17}$, we further examined EXPLORs containing undetectable amounts of plasmid DNA (data not shown) and mCherry-CRY2 mRNA (Supplementary Fig. 9b). In addition, we tested the loading efficiency of mCherry proteins in EXPLORs in response to cycles of blue light illumination and darkness. Using a variety of cycle combinations, we identified a cycle consisting of $1 \mathrm{~min}$ of blue light illumination followed by $1 \mathrm{~min}$ of darkness as the optimal condition to produce mCherry:EXPLORs (Supplementary Fig. 10).

We next examined the loading capacity of EXPLOR technology by producing firefly luciferase-containing EXPLORs. For the production of luciferase:EXPLOR, we cloned vectors for firefly luciferase-conjugated mCherry-CRY2 and confirmed lightinduced PPI with CIBN-EGFP-CD9 by blue light stimulation with confocal microscopy (Supplementary Fig. 11). First, we compared luciferase:EXPLORs with a commercialized method for exosome protein loading, XPACK (System Biosciences, Mountain View, CA, USA), which is based on the principle that highly oligomeric, cytoplasmic proteins can be targeted to exosomes by plasma membrane anchors ${ }^{18,19}$. We also included exosomes produced from cells transfected with the luciferase-conjugated mCherry-CRY2 vector, but not with the CIBN-EGFP-CD9 expression plasmid, to exclude the possibility of nonspecific loading of overexpressed cargo proteins into exosomes. Transfection efficiency and expression profile of luciferasemCherry fusion proteins were comparable between the different methods (Fig. 3a,b and Supplementary Fig. 12). For the quantitative analysis of cargo protein loading into the 
a

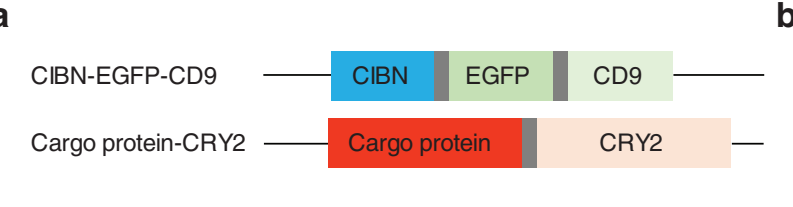

C
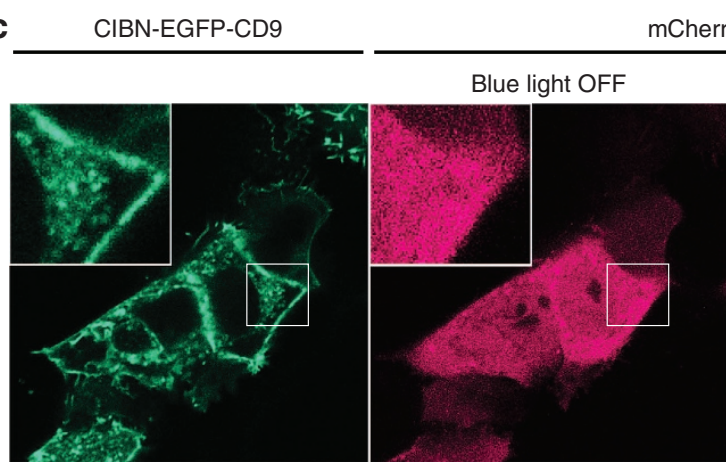

mCherry-CRY2
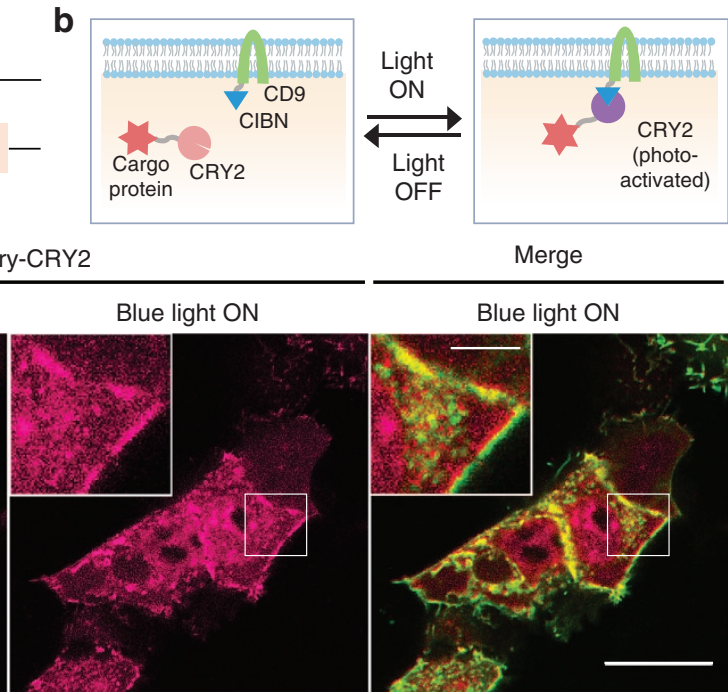

Merge

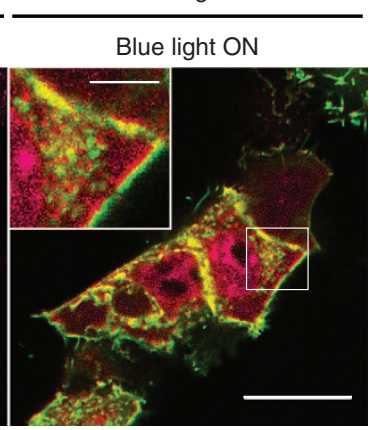

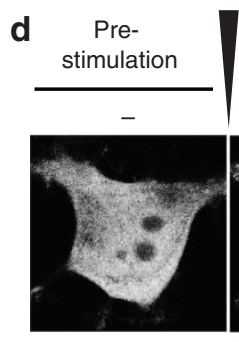
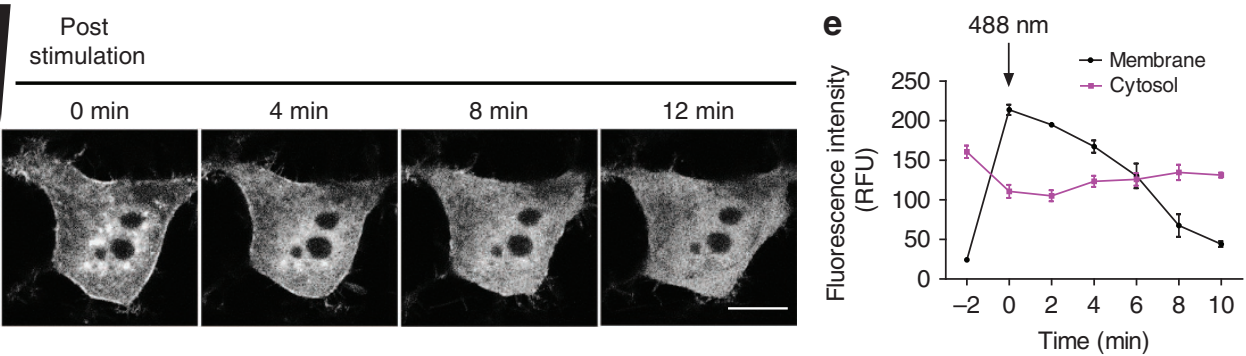

Figure 1 | Generation of engineered EXPLOR. (a) Schematics of DNA constructs used for the production of EXPLOR. (b) Schematic showing fusion proteins and their proposed action. (c) HEK293T cells were transiently transfected with CIBN-EGFP-CD9 and mCherry-CRY2 expression vectors. The mCherry fluorescence was imaged before and after $488-\mathrm{nm}$ laser stimulation ( $15 \mathrm{~s}$ in duration, $350 \mu \mathrm{W} \mathrm{cm}^{-2}$ ). Scale bars, $20 \mu \mathrm{m}$ ( $5 \mu \mathrm{m}$ for inset images). A representative result from at least 10 experiments. (d) HEK293T cells transiently transfected with CIBN-EGFP-CD9 and mCherry-CRY2 were imaged for time-lapse imaging of mCherry fluorescence for varying time periods ( $0-12 \mathrm{~min}$ ) after a stimulation (black arrow) of $488-\mathrm{nm}$ light (15 $\mathrm{s}$ in duration, $350 \mu \mathrm{W} \mathrm{cm}^{-2}$ ). Scale bars, $5 \mu \mathrm{m}$. A representative result of at least 10 experiments. (e) Quantification of mCherry fluorescence in the cytoplasm and at the plasma membrane. Data are presented as the mean \pm s.e.m. $(n=3)$.

a

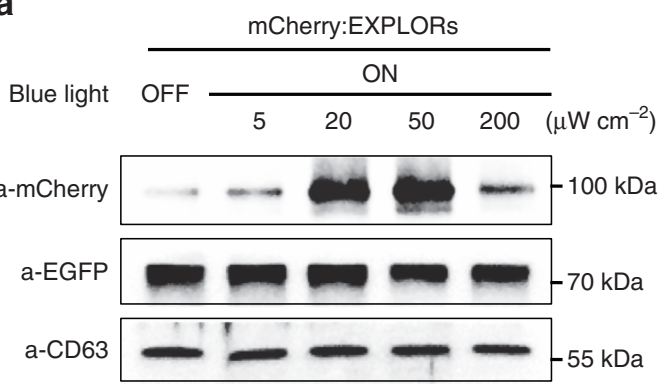

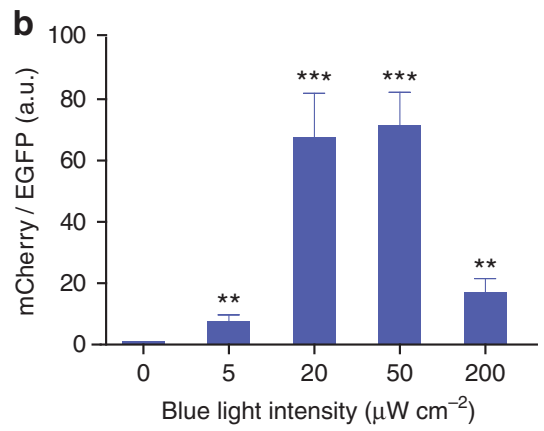

Blue light intensity $\left(\mu \mathrm{W} \mathrm{cm}{ }^{-2}\right)$

Figure 2 | Light-dependent loading of target proteins in EXPLORs. (a) Cells transiently transfected with CIBN-EGFP-CD9 and mCherry-CRY2 expression vectors were maintained under blue light illumination of varying powers for $48 \mathrm{~h}$. Cell-derived exosomes were subject to immunoblot analysis using antibodies against mCherry, EGFP and CD63, an exosome marker. A representative result from three independent experiments. (b) The graph presents densitometry analysis for normalized amount of mCherry-CRY2 protein over CIBN-EGFP-CD9 protein from three independent experiments. Data are presented as the mean \pm s.e.m. $(n=3)$, and Tukey's post hoc test was applied to significant group effects $\left({ }^{\star \star} P<0.01\right.$, $\left.{ }^{\star \star \star} P<0.001\right)$ identified by analysis of variance.

exosomes, we measured luciferase activity using the same numbers of isolated exosomes (Fig. 3c,d). The results showed that the loading capacity of EXPLORs was significantly higher than those of the overexpression model of luciferase-mCherryCRY2 and a commercialized model for exosome protein targeting. In our experiment, it was estimated that, on average, 1.4 molecules of luciferase proteins were included in each exosome obtained by EXPLOR technology (Supplementary Fig. 13). We also tested another method: ex vitro protein loading in naive exosomes loaded with recombinant luciferases. In total, $50 \mu \mathrm{g}$ of recombinant luciferases $(61 \mathrm{kDa})$ dissolved in phosphate-buffered saline (PBS) was added to $2 \times 10^{10}$ exosomes derived from HEK293T cells and loaded into exosomes ex vitro with an extrusion method ${ }^{20}$. Only $4.4 \mathrm{pg}$ of recombinant protein 


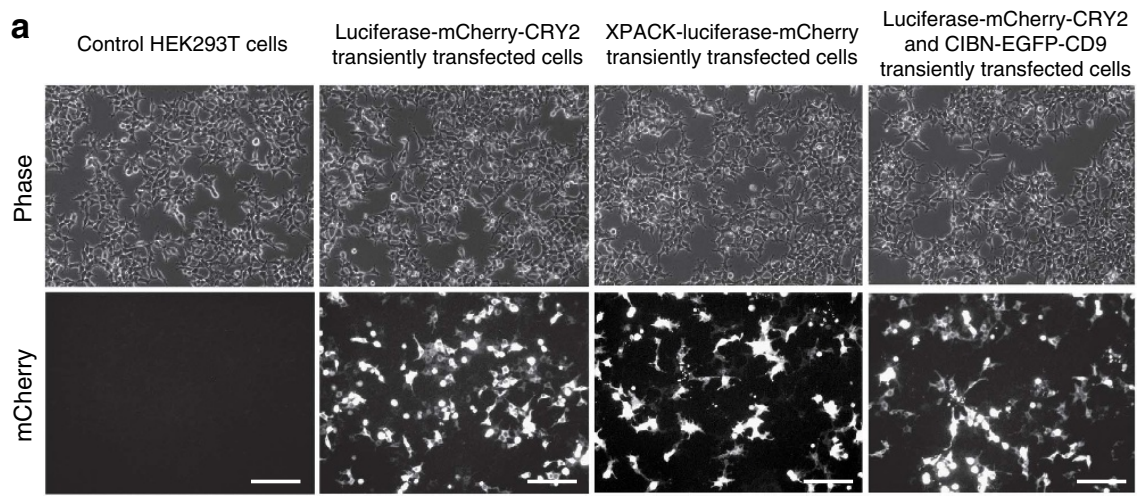

b
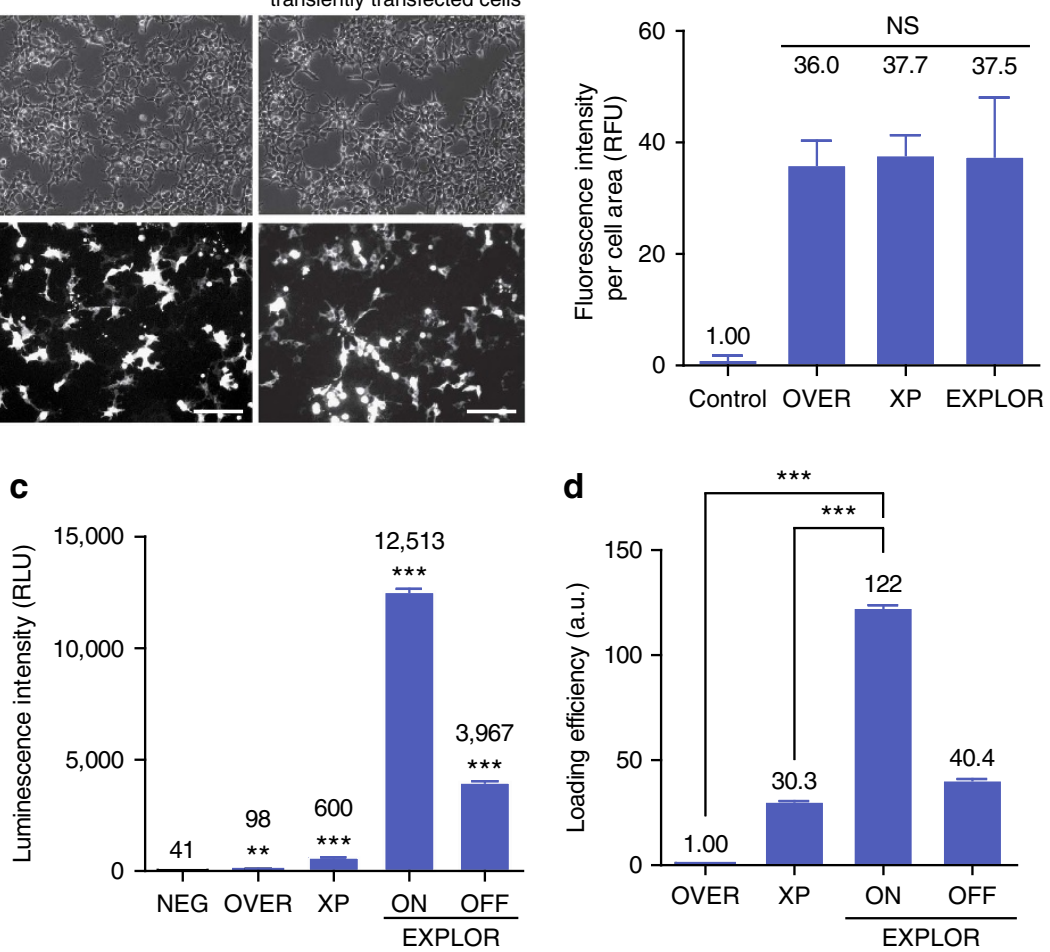

d

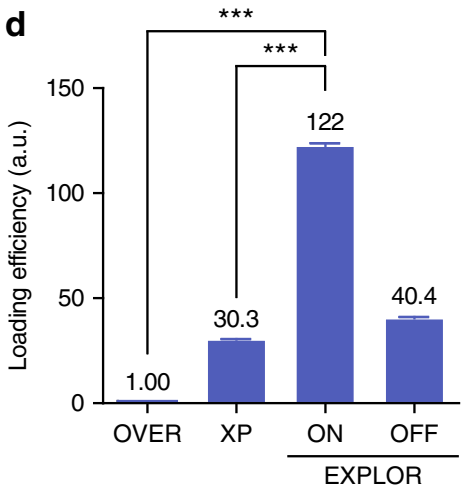

NEG negative exosomes

OVER exosomes from luciferase-mCherry-CRY2 transiently expressing cells

XP exosomes from XPACK-luciferasemCherry transiently expressing cells

EXPLOR luciferase-mCherry:EXPLORs

(ON) produced under blue light $\mathrm{ON}$ condition (OFF) produced under light OFF condition

Figure 3 | Comparison of the exosome-loading capacity of target proteins between various protein-loading methods. (a) HEK293T cells were transiently transfected with luciferase-mCherry-CRY2 expression vector alone, XPack-luciferase-mCherry expression vector or co-transfected with CIBN-EGFPCD9 and luciferase-mCherry-CRY2 expression vectors. After $24 \mathrm{~h}$, cells were imaged by fluorescence microscopy for the expression profile of mCherry fusion proteins. A representative result from five independent experiments. Scale bars, $20 \mu \mathrm{m}$. (b) Quantification of mCherry fluorescence. Data are presented as the mean \pm s.e.m. $(n=5)$, and Tukey's post hoc test was applied to significant group effects identified by analysis of variance (ANOVA). Control: untransfected HEK293T cells; OVER: cells transiently transfected with a luciferase-mCherry-CRY2 vector; XP: cells transfected with an XPACK-luciferase$m$ Cherry vector; and EXPLOR: cells transfected with both luciferase-mCherry-CRY2 and CIBN-EGFP-CD9 vectors. (c) Cells transiently transfected with various vectors were maintained for $48 \mathrm{~h}$. In the case of EXPLOR-producing cells, cells were maintained in the absence (OFF) or presence (ON) of blue light illumination; $5 \times 10^{8}$ particles of the isolated exosomes were analysed for luciferase activity. Data are presented as the mean \pm s.e.m. ( $n=3$ ), and Tukey's post hoc test was applied to significant group effects $\left({ }^{\star \star} P<0.01,{ }^{\star \star \star} P<0.001\right)$ identified by ANOVA. (d) Loading efficiency of various protein-loaded exosomes was calculated by dividing the number of luciferase molecules in exosomes with the number of luciferase molecules in the exosome-producing cells. Numbers of luciferase molecules were estimated from a standard curve using recombinant luciferase. Data are presented as the mean \pm s.e.m. $(n=3)$, and Tukey's post hoc test was applied to significant group effects $\left({ }^{\star \star} P<0.001\right)$ identified by ANOVA. NS, not significant.

was finally included in the exosomes; thus, the loading capacity of the ex vitro protein-loading method was estimated to be 40 -fold lower than that of the EXPLOR technology (Supplementary Fig. 14).

Administration of EXPLORs in vitro and in vivo. HeLa cells were incubated in the absence or presence of mCherry-loaded exosomes for $24 \mathrm{~h}$, and tested for analysis with EXPLORmediated protein delivery to recipient cells (Fig. 4a). Consistent with previous results, EXPLOR-treated cells showed significantly higher fluorescence signals (Fig. 4b). Cryo-immunogold electron microscopy analysis (Supplementary Fig. 15a) and confocal microscopy (Supplementary Fig. 15b) revealed that mCherry proteins were clearly transferred into the cytosolic region of cells after incubation with mCherry:EXPLORs derived under the 'light ON' condition. Immunoblot analysis demonstrated significant increases in the amounts of intracellular mCherry proteins in recipient cells after treatment with mCherry:EXPLORs in both a time- and dose-dependent manner (Supplementary Fig. 15c,d).

To further confirm whether EXPLOR technology can be useful for the delivery of functional proteins, we cloned vectors for mCherry-CRY2 proteins fused with Bax and super-repressor I $\mathrm{B}$ $(\operatorname{srI\kappa B})$ proteins (Supplementary Fig. 16). In the normal condition, Bax proteins are mainly distributed in the cytosol; however, on initiation of the apoptotic process, they undergo a conformation change and move to the mitochondrial membrane, leading to the release of cytochrome $c$ that subsequently triggers caspase-dependent apoptosis ${ }^{21-24}$. As expected, incubation with Bax-mCherry:EXPLORs induced a rapid release of cytochrome $c$ from the mitochondria in HeLa cells (Fig. 4c,d). Similarly, the endogenous nuclear factor $-\kappa \mathrm{B}(\mathrm{NF}-\kappa \mathrm{B})$ inhibitor $\mathrm{I} \kappa \mathrm{B}$ proteins bind to the NF- $\kappa \mathrm{B}$ complex in the cytoplasm; however, on relevant stimulation, $I \kappa \mathrm{B}$ is phosphorylated by $\mathrm{I} \kappa \mathrm{B}$ kinases and degraded by the ubiquitin-proteasome pathway. Then, the NF- $\kappa \mathrm{B}$ complexes translocate to the nuclei and induce the expression of survival- and inflammation-related genes ${ }^{25}$.

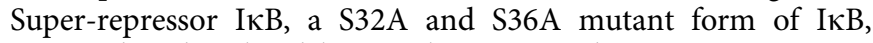
is not phosphorylated by $I \kappa B$ kinases, so that $s r I \kappa B$ suppresses the translocation of NF- $\kappa \mathrm{B}$ complex even in the presence of pro-inflammatory stimulation ${ }^{26}$. As expected, treatment with srIкB-mCherry:EXPLORs significantly reduced tumour necrosis factor- $\alpha$-induced translocation of the p65 subunit of NF- $\kappa B$ in HeLa cells (Fig. 4e,f).

Finally, we validated the potential of EXPLOR-mediated intracellular protein delivery for in vivo application. To test the intracellular delivery of Cre recombinase containing EXPLORs 
a

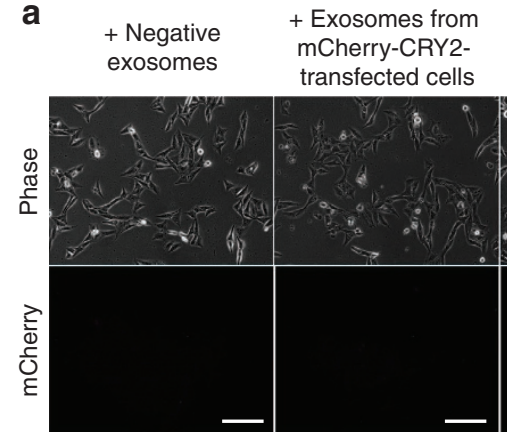

C
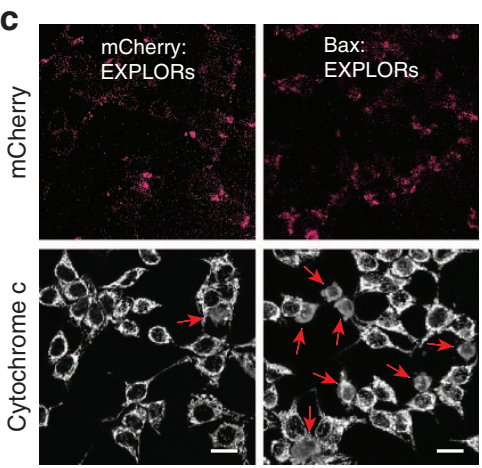

+ Exosomes from XPACK-mCherry
+ mCherry:EXPLORs

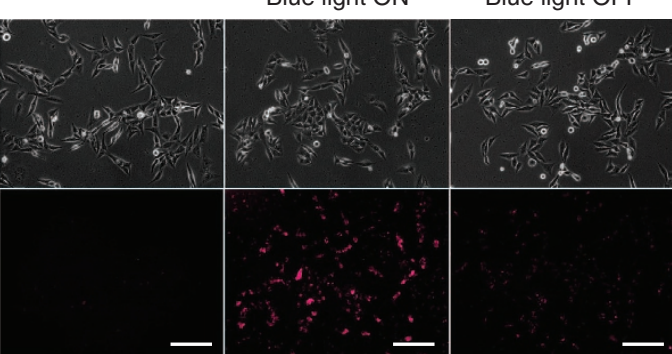

d Mitochondria

Cytosol

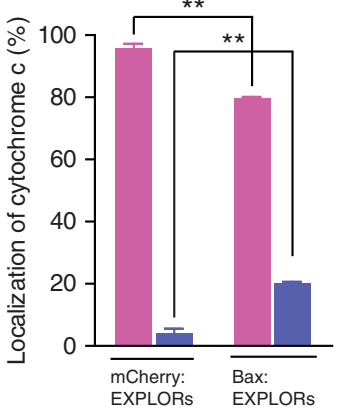

e

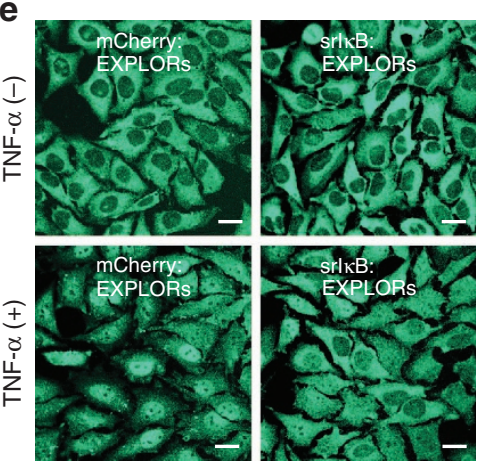

b

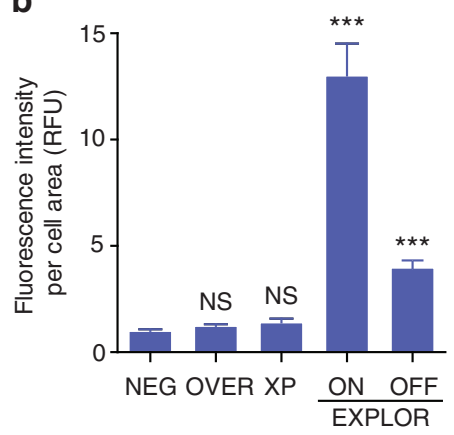

f

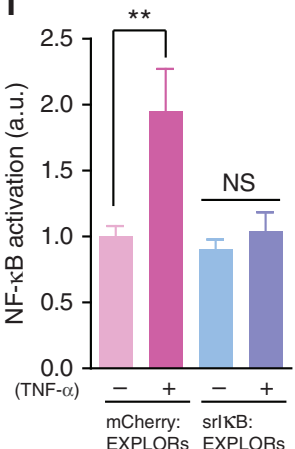

Figure 4 | EXPLOR-mediated intracellular delivery of cargo proteins. $(\mathbf{a}, \mathbf{b})$ HeLa cells were incubated in the absence or presence of $5 \times 10^{9}$ particles of various isolated exosomes for $24 \mathrm{~h}$ and imaged by fluorescence microscopy. The fluorescence intensities of mCherry were quantified by two imaging processing tools, ImageJ and Cellprofiler. Data are presented as the mean \pm s.e.m. $(n=15)$, and Tukey's post hoc test was applied to significant group effects $\left.{ }^{\star \star \star} P<0.001\right)$ identified by analysis of variance (ANOVA). Scale bars, $100 \mu \mathrm{m}$. (c,d) HeLa cells were incubated in the absence or presence of $0.1 \mathrm{mg} m \mathrm{ml}^{-1}$ mCherry:EXPLORs or Bax-mCherry:EXPLORs for $12 \mathrm{~h}$, and fixed with $4 \%$ paraformaldehyde. Then, cytochrome $c$ was stained with an antibody conjugated with Alexa Fluor 647 and imaged by confocal microscopy. The ratios of cytochrome $c$ localization were analysed by cell counting. Data are presented as the mean \pm s.e.m. $(n=3)$, and Tukey's post hoc test was applied to significant group effects ( $\left.{ }^{\star} P<0.01\right)$ identified by ANOVA. Scale bars, $20 \mu \mathrm{m}$. (e) HeLa cells were incubated in the absence or presence of $0.1 \mathrm{mg} \mathrm{ml}^{-1} \mathrm{mCherry:EXPLORs} \mathrm{or} \mathrm{srl \kappa B:EXPLORs} \mathrm{for} 12 \mathrm{~h}$, treated with $10 \mathrm{ng} \mathrm{ml} \mathrm{m}^{-1}$ tumour necrosis factor- $\alpha$ $(\mathrm{TNF}-\alpha)$ for an additional $30 \mathrm{~min}$, and fixed with $4 \%$ paraformaldehyde. NF- $\mathrm{kB}$ p 65 was stained with an antibody conjugated with Alexa Fluor 488 and imaged by confocal microscopy. (f) The nuclear extracts of cells were assayed for the DNA-binding activity of p65/c-Rel (NF- $\kappa B$ ). Data are presented as the mean \pm s.e.m. $(n=3)$, and Tukey's post hoc test was applied to significant group effects $\left({ }^{\star \star} P<0.01\right)$ identified by ANOVA. Scale bars, $20 \mu \mathrm{m}$. NS, not significant.

(Cre:EXPLORs), we cloned a vector for Cre-conjugated mCherryCRY2 (Cre-mCherry-CRY2) protein. Unlike mCherry-CRY2 protein, Cre-mCherry-CRY2 proteins were predominantly expressed in the nuclei, and blue light illumination induced limited translocation of these proteins to the plasma membrane and intracellular endosomal fractions (Supplementary Fig. 17a). To avoid unwanted nuclear localization and increase the loading efficiency of Cre-mCherry-CRY2 proteins, cells were maintained under blue light illumination immediately after transfection. As expected, continuous light illumination immediately after transfection markedly enhanced co-localization of Cre-mCherryCRY2 proteins with CIBN-EGFP-CD9 (Supplementary Fig. 17b). The enzymatic activity of Cre recombinase was validated in the target cells previously transfected with a reporter plasmid containing a loxP-STOP-loxP sequence upstream of the ZsGreenencoding gene. Treatment with Cre:EXPLORs clearly induced green fluorescence at levels comparable to that of transfection of pCMV-Cre vectors in various cells including rat embryonic primary neurons (Supplementary Fig. 18). Because the transient transfection of primary neurons is extremely inefficient, we next tested the effect of Cre:EXPLORs on neurosphere-derived differentiated neuronal cells isolated from transgenic mice having a loxP-STOP-loxP-EGFP gene. As expected, treatment with Cre:EXPLORs induced EGFP expression in over $95 \%$ of cells; while treatment with naive EXPLORs had no effect (Fig. 5a,b). Next, we injected Cre:EXPLORs into the ventrolateral part of the brain of
pCAG-loxP-STOP-loxP-eNpHR3.0-EYFP (enhanced yellow fluorescence protein) transgenic mice (Fig. 5c). After $96 \mathrm{~h}$, eNpHR3.0 EYFP proteins were expressed widely near the ventrolateral part in the brain, and especially were expressed highly in the zona incerta region (Fig. 5d). Immunohistochemical analyses demonstrated that the EYFP-positive cells were mainly neurons, indicating that Cre:EXPLORs worked functionally mainly in neurons in vivo (Fig. 5e and Supplementary Fig. 19).

\section{Discussion}

Although protein transduction and lipid nanoparticle-mediated protein delivery methods have been proposed for direct protein delivery into target cells and tissues $6,7,27$, many obstacles remain before these methods can be successfully used in vivo, including low purification efficiency, failure to separate from nanoparticles in recipient cells and induction of immune responses against host immune cells. To address these limitations, we developed an exosome-based delivery system EXPLORs that can be readily produced using live cells as a factory via the endogenous biogenesis of extracellular vesicles (Fig. 6).

This novel method provides significant advantages for both research and clinical applications. First, EXPLORs can carry cargo proteins without isolation or purification of recombinant proteins. Because EXPLORs contain cell-produced cargo proteins in unbound soluble form, this approach eliminates many of the issues such as misfolding of recombinant proteins or separation 

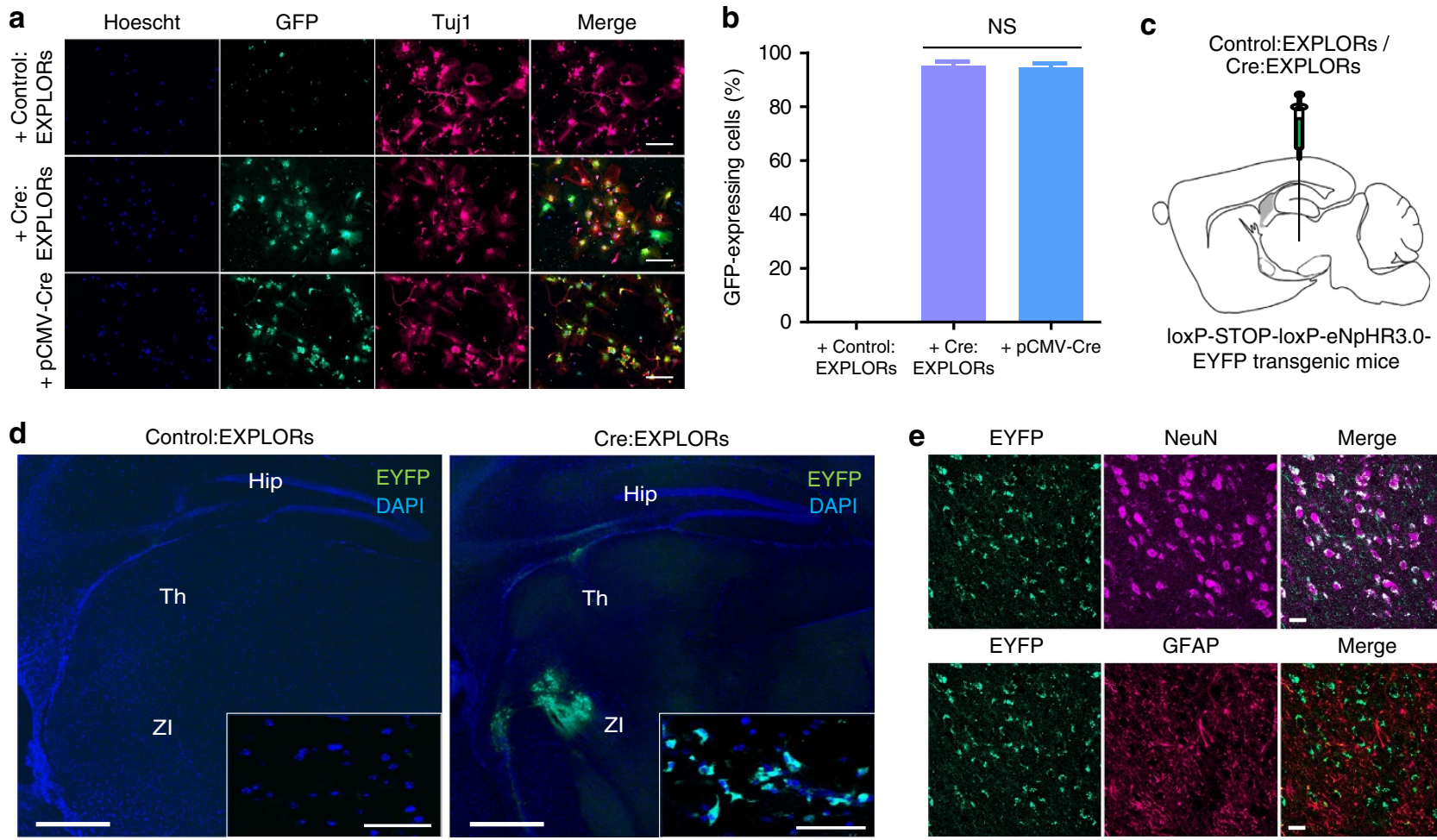

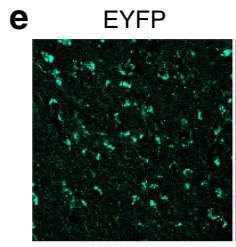

EYFP

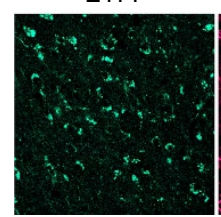

NeuN

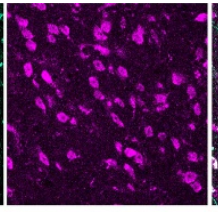

GFAP

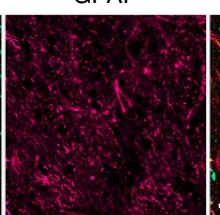

Merge

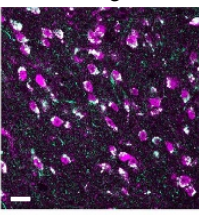

Merge

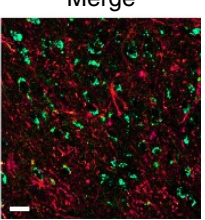

Figure 5 | EXPLOR-mediated delivery of Cre recombinase in vitro and in vivo. (a,b) Differentiated neurosphere-derived cells were incubated in the absence or presence of $2 \times 10^{10}$ particles per $\mathrm{ml}$ of Cre:EXPLORs $\left(0.16 \mathrm{mg} \mathrm{ml}^{-1}\right)$ or transfected with $p C M V$-Cre vector for $72 \mathrm{~h}$. Cells were fixed with $4 \%$ paraformaldehyde and immune-stained with antibodies against a neuron-specific class III beta-tubulin marker, Tuj1, GFP and Hoechst 33342. The ratios of EGFP-expressing cells were analysed by cell counting. Data are presented as the mean \pm s.e.m. $(n=10$ fields), and Tukey's post hoc test was applied to significant group effects identified by ANOVA. Scale bars, $100 \mu \mathrm{m}$. (c) An experimental scheme for the administration of Cre:EXPLORs in loxp-stop-loxpeNpHR3.0-EYFP transgenic mice. In total, $50 \mu$ l of Cre:EXPLORs $\left(10 \mathrm{mg} \mathrm{ml}^{-1}\right.$ ) were administered to $p$ CAG-loxP-STOP-loxP-eNpHR3.0-EYFP transgenic mice by ventrolateral injection. (d) Brain slices of EXPLOR-injected transgenic mice were fixed with $4 \%$ formaldehyde and imaged by fluorescence microscopy. Green fluorescence indicates eNpHR3.0-EYFP protein expression and blue fluorescence indicates cell nuclei. Inset images showed confocal microscopy images of the detailed cellular eNpHR3.0-EYFP expression in EXPLOR-administered mouse neurons of the zona incerta (ZI) region. Scale bars, $500 \mu \mathrm{m}$ ( $50 \mu \mathrm{m}$ for inset confocal images). Hip, hippocampus; Th, thalamus. A representative of two independent experiments. (e) Representative image of NeuN/GFAP immunohistochemistry of the brain. Pink, neuronal-specific nuclear protein (NeuN)-positive neurons; Red, glial fibrillary acidic protein (GFAP)-positive astrocyte cells. Objective lens, $\times 40$. Scale bar, $20 \mu \mathrm{m}$. NS, not significant.

of nanoparticles and cargo proteins in the target cells. Second, EXPLORs can be readily and easily produced once the appropriate EXPLOR-producing donor cells have been selected from genetically engineered cells. By simply irradiating with a blue light, we can generate EXPLORs loaded with specific cargo proteins, including those derived from other cell types. This stable production of EXPLORs from genetically engineered cells has great potential for the commercialization of standardized exosome-based protein therapeutics. Finally, EXPLORs are compatible with many personalized medicine-based therapeutic approaches. By modifying patient-derived cells to produce EXPLORs $^{28}$, we would be able to manufacture patientcustomized drug delivery systems with reduced risk of triggering host immune responses, opening a new paradigm for personalized protein-based therapeutics.

In the present study, we showed that exosomes from cells transiently transfected with a luciferase-mCherry-CRY2 vector rarely had luciferase molecules; however, EXPLORs that were derived from cells transfected with luciferase-mCherry-CRY2 and $C I B N-E G F P-C D 9$ vectors under the light OFF condition had considerable numbers of luciferase molecules. The difference between the two conditions was CIBN-EGFP-CD9 expression. Recently, one research group showed that overexpressed CRY2-conjugated proteins have a weak binding activity with CIBN-conjugated proteins in the normal state ${ }^{29}$. Thus, we suggest that unintended binding of mCherry-CRY2 to CIBN-EGFP-CD9 could be partially responsible for loading of mCherry proteins into exosomes under the light OFF condition.

EXPLORs had the highest efficiency in protein encapsulation under blue light illumination in the power range of $20-50 \mu \mathrm{W} \mathrm{cm}^{-2}$, not at the strongest power $\left(200 \mu \mathrm{W} \mathrm{cm}{ }^{-2}\right)$. We found that incubation of cells under blue light illumination at a power of $200 \mu \mathrm{W} \mathrm{cm}{ }^{-2}$ did not induce significant cell death; thus, cell death was not the cause of decreased protein loading efficiency according to strong light. We presumed that aggregation of CRY2 proteins by strong blue light might disturb the light-induced PPI between mCherry-CRY2 and CIBN-EGFPCD9 proteins $^{16}$. To address this, we used CRY2 fusion proteins conjugated with Cre recombinase. Unlike other CRY2 fusion proteins conjugated with cytosolic proteins, Cre-mCherry-CRY2 proteins were expressed mainly in the nuclei. Because aggregated forms of Cre-CRY2 proteins cannot diffuse readily through nuclear membranes, we can readily study the effect of light intensity on the aggregation of CRY2 proteins by simply analyzing the subcellular localization of Cre-mCherry-CRY2 proteins. As expected, we observed that the stronger the blue light illuminated, the more Cre-mCherry-CRY2 stayed in the cytosolic compartment, rather than inside the nucleus, confirming that CRY2-fusion proteins can form aggregates under high-intensity light conditions (Supplementary Fig. 20). 

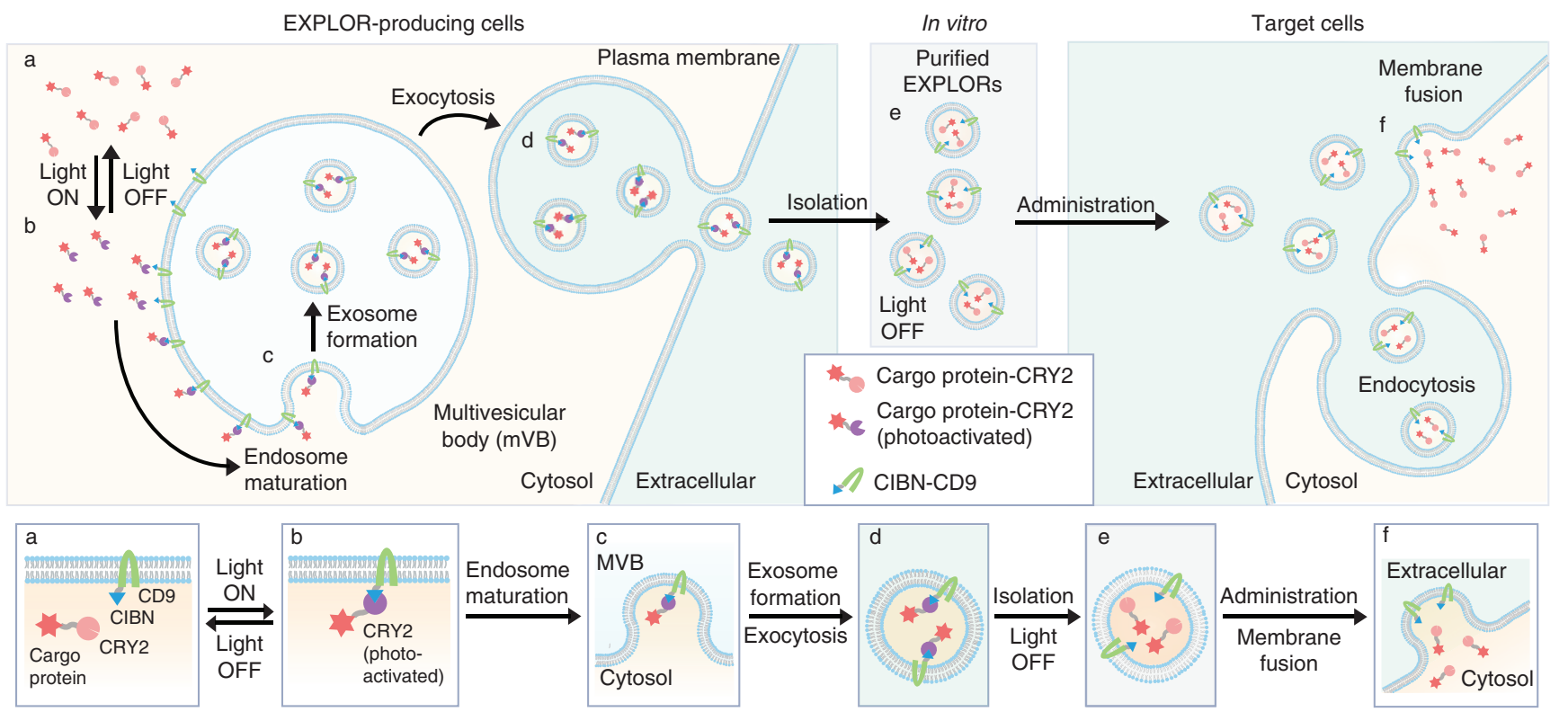

Figure 6 | Schematic diagram of EXPLOR technology. In EXPLOR-producing donor cells, CRY2 protein was fused to a cargo protein, and CIBN was conjugated with a representative marker of exosomes, CD9 protein. Blue light illumination induces the reversible PPI between CIBN and CRY2 fusion proteins. With continuous blue light irradiation, the cargo proteins are guided to the inner surface of the cell membrane or the surface of early endosomes. Mature multi-vesicular bodies (MVBs) then readily secrete cargo protein-carrying exosomes (EXPLORs) from the cells by membrane fusion with the plasma membrane. After exocytosis, EXPLORs can be easily isolated and purified in vitro. Purified EXPLORs can be used for delivery of the cargo proteins into target cells via membrane fusion or endocytosis processes. Bottom grey boxes highlight the essential steps from EXPLORs biogenesis to target cell delivery.

We found that one or two cargo molecules were contained in one particle of EXPLOR on average; however, this limited loading efficiency might still have room for improvement. In the present study, we used fusion proteins of fluorescence reporters with both the CIBN and CRY2 protein for better tracking of target proteins. Because we have confirmed that target proteins conjugated with CRY2 can be successfully recruited to engineered exosomes, direct conjugation of cargo proteins or tetraspanins to CRY2 or CIBN proteins without fluorescent reporter proteins can further decrease the molecular size of cargo proteins and subsequently improve loading efficiency of cargo proteins into EXPLORs. In addition, the current strategy using two vectors can further be optimized by using a one-vector system, by introducing an internal ribosome entry site. These approaches might increase the transfection efficiency, as well as the loading of cargo proteins into EXPLORs.

In the present study, we developed a novel protein carrier EXPLOR that has a higher loading capacity and delivery efficiency than previous methods for protein-loaded exosomes. Also, we demonstrated the intracellular delivery of mCherry, Bax, super-repressor I $\mathrm{I} B$ protein and Cre enzyme as functional proteins into the target cells in vitro and into brain parenchymal cells in vivo. Many intracellular proteins, such as transcription factors, signal transducers and enzymes can also be attractive targets for EXPLOR-based therapeutics. Using our EXPLOR technique, we might be able to design various EXPLOR-producing cells that would be equivalent to monoclonal antibody-producing hybridoma cells for protein-based therapeutics targeting intracellular events.

\section{Methods}

Cell culture. HEK293T human embryonic kidney cells (CRL-3216, ATCC, Manassas, VA, USA), HT1080 cells (CCL-121, ATCC) and HeLa cells (CCL-2, ATCC) were maintained in Dulbecco's modified Eagle's medium (DMEM; Welgene, Seoul, Korea) containing 10\% fetal bovine serum (FBS; Gibco, Gaithersburg, MD, USA) and $1 \%$ penicillin-streptomycin (Gibco).
Blue LED-installed incubator. The 460-nm LED bars for plant growth (TG-TECH, Asan, Korea) were installed in a $\mathrm{CO}_{2}$ incubator for illuminating cells with blue light. For controlling the light power of the blue LED, a LED dimmer (Hyeonju LED, Seoul, Korea) was added. Automatic control of the on/off cycle was achieved using an infinite-iteration timer attached to the power source (Seojun Electronics, Seoul, Korea). To equally illuminate blue light on the cells, a polycarbonate plate of $3 \mathrm{~mm}$ width (BEST ACRYLIC, Incheon, Korea) was added under the blue LED bars as a light diffuser. An optical power meter $8230 \mathrm{E}$ (ADCMT, Tokyo, Japan) was used to assess the LED illumination power.

Reagents. Primary antibody sources were as follows: mouse monoclonal mCherry antibody (diluted 1:1,000 for western blotting, 1:50 for EM, ab125096), rabbit monoclonal CD9 antibody (diluted 1:50 for EM, ab92726) and NF- $\mathrm{\kappa B}$ antibodies (diluted 1:1,000 for immunocytochemistry, ab16502) were obtained from Abcam (Cambridge, MA, USA); CD63 (diluted 1:1,000 for western blotting, sc-15363) and GAPDH (diluted 1:4,000 for western blotting, sc-25778) were purchased from Santa Cruz Biotechnology (Santa Cruz, CA, USA); cytochrome $c$ antibody (diluted 1:1,000 for immunocytochemistry, 556432) was from BD biosciences (San Jose, CA, USA); and the GFP antibody (diluted 1:1,000 for western blotting, 2555) was from Cell Signaling Technology (Beverly, MA, USA). Secondary antibody sources were as follows: anti-rabbit (sc-2004) and mouse secondary antibody (sc-2005) were obtained from Santa Cruz Biotechnology, and Alexa Fluor 647 goat anti-mouse IgG $(\mathrm{H}+\mathrm{L})$ antibody (diluted 1:1,000 for ICC, A-21235) and Alexa Fluor 488 goat anti-rabbit IgG $(\mathrm{H}+\mathrm{L})$ antibody (diluted 1:1,000 for ICC, A-11008) were purchased from Thermo Fisher Scientific (Wilmington, DE, USA).

Plasmids and transfection. All plasmids and PCR primers used in this study are listed in Supplementary Table 2. $p C M V-C I B N-E G F P$ and $p C M V$ - $m$ Cherry-CRY2 (codon-optimized for expression in mammalian cells) were generated ${ }^{30}$. To generate the $p C M V$-CIBN-EGFP-CD9 construct, a sequence encoding full-length $C I B N-E G F P$ was PCR-amplified from CIBN-EGFP constructs, and a gene encoding CD9 was PCR-amplified from the LN215 cDNA library using gene-specific primers. For the CIBN-EGFP-CD9 construct, the first fragment (CIBN-EGFP-) contained an EcoRI site, a sequence encoding the full-length CIBN-EGFP, and a sequence encoding a flexible linker (SGGGGSGGGGSGGGGS). The second PCR fragment contained a flexible linker, a sequence encoding full-length $C D 9$, and a XhoI site, followed by 15 base pairs of homology to the pcDNA3.1 $(+)$ (Invitrogen, Carlsbad, CA, USA) vector. To construct exosomal membrane-targeting sequence-tagged mCherry, the $m$ Cherry-encoding gene was amplified from an mCherry-CRY2 vector and ligated into an EcoRI site of XPack CMV-XP-MCSEF1-Puro Cloning Lentivector (System Biosciences, Mountain View, CA, USA). To assemble $p C M V$-Cre-mCherry-CRY2, genes encoding Cre were PCR-amplified 
from the $p C M V$-Cre vector (Addgene plasmid 11916) using gene-specific primers. For the construct, the first fragment (Cre-) contained an EcoRI site, a sequence encoding full-length Cre except a stop codon and a sequence encoding a flexible linker (SAGGPPVAT). The second PCR fragment ( $m$ Cherry-CRY2) contained the flexible linker, a sequence encoding full-length $m$ Cherry-CRY2 and a Xhol site, followed by 15 base pairs with homology to the pcDNA3.1 $(+)$ vector. A pCAG-loxP-STOP-loxP-ZsGreen plasmid was obtained from Addgene (plasmid 51269). The Bax gene was amplified from the LN215 cDNA library, super-repressor IKB was obtained from Addgene (plasmid 15294) and firefly luciferase gene was obtained from the $p G L 4.11[l u c 2 P]$ vector (Promega, Madison, WI, USA). Cells were transfected with Effectene Transfection Reagent (Qiagen, Valencia, CA, USA) at a density of $50-80 \%$ confluence, according to the manufacturer's protocol

Microscopy for cell imaging. Live cells were imaged using a Zeiss LSM 510 confocal microscope with a $\times 63$ Apochromat 1.0 numerical aperture (NA) objective (Carl Zeiss, Thornwood, NY, USA). Laser light $(488 \mathrm{~nm})$ was used to induce dimerization between CRY2PHR and CIBN and to image GFP fusion proteins; a 594- $\mathrm{nm}$ laser was used to image mCherry-fusion proteins. $Z$-stack and time-lapse imaging were also performed using the confocal microscope, and the three-dimensional reconstruction and real-time images were processed using ImageJ software. An environmental chamber (Harvard Apparatus, South Natick, MA, USA) enclosing the microscope stand was used to maintain the temperature at $37^{\circ} \mathrm{C}$ with $5 \% \mathrm{CO}_{2}$ during live cell imaging. For fluorescence microscope imaging, cells were fixed with $4 \%$ paraformaldehyde at $30 \mathrm{~min}$ and imaged using a Zeiss Axiovert $200 \mathrm{M}$ fluorescence imaging microscope with $\times 10$ Plan-NeoFluar 0.3 NA objective and a $20 \times$ Plan-NeoFluar 0.5 NA objective (Carl Zeiss). The quantification of cell fluorescence was analysed using the Cellprofiler software (Broad Institute, Cambridge, MA, USA).

Exosome isolation. Cells were collected and transferred to fresh FBS-free DMEM (Welgene) containing $1 \%$ penicillin-streptomycin (Gibco) or DMEM containing $1 \%$ penicillin-streptomycin and $10 \%$ exosome-depleted FBS ${ }^{31}$. After $48 \mathrm{~h}$, medium was collected and centrifuged at $3,000 \mathrm{~g}$ for $15 \mathrm{~min}$ to remove cellular debris. Exosome isolation was performed by precipitation or ultracentrifugation. For precipitation, the supernatant was transferred to a sterile vessel and the appropriate volume of ExoQuick-TC Exosome Precipitation Solution (System Biosciences) was added. Samples were mixed by inverting or flicking the tube and then refrigerated overnight. After $24 \mathrm{~h}$, the ExoQuick-TC mixture was centrifuged (1,500g, $30 \mathrm{~min}$ ). After centrifugation, the supernatant was removed and the mixture was spun down again $(1,500 \mathrm{~g}, 5 \mathrm{~min})$. All traces of fluid were removed by aspiration, and the exosome pellet was resuspended in $100 \mu \mathrm{l}$ PBS by passaging 5-10 times through a sterile 27-gauge needle (BD Biosciences, San Jose, CA, USA). Then, the suspended exosomes were filtered through a syringe filter $(0.2 \mu \mathrm{m}$; Sartorius, Goettingen, Germany) to prevent aggregation of exosomes and to remove Exoquick polymers. For ultracentrifugation-based purification of exosomes, supernatants were transferred to an appropriate vessel for the MF-600 ultracentrifuge (Hanil Science Industrial, Incheon, Korea) and centrifuged $(120,000 g, 2 \mathrm{~h})$. After centrifugation, all traces of fluid were removed by aspiration, and the exosome pellet was resuspended in $100 \mu \mathrm{l}$ PBS by passaging 5-10 times through a sterile 27-gauge needle (BD Biosciences). The suspended exosomes were then filtered through a syringe filter $(0.2 \mu \mathrm{m}$, Sartorius). The concentration of suspended exosomes was measured using a Nanodrop ND-1000 spectophotometer (Thermo Scientific, Wilmington, DE, USA). The size of the suspended exosomes was measured with a Zetasizer ZS90 dynamic light scattering machine, operating in intensity mode using a $640-\mathrm{nm}$ HeNe laser (Malvern Instruments, Worcestershire, UK). The number of exosome particles was measured by nanoparticle-tracking analysis with a Nanosight NS300 (Malvern Instruments).

Immunoblotting. Collected cells or isolated exosomes were washed in ice-cold PBS, and suspended in adjusted volumes of buffer, provided with the M-Per mammalian protein extraction kit (Pierce, Rockford, IL, USA). The cells were incubated on ice for $30 \mathrm{~min}$, and the lysates were then cleared by centrifugation at $10,000 \mathrm{~g}$ for $10 \mathrm{~min}$. Lysates were then separated by $12 \%$ SDS-polyacrylamide gel electrophoresis, transferred to nitrocellulose membranes (Invitrogen, Carlsbad, CA, USA), and incubated with the appropriate antibodies. The immunoblots were imaged using an enhanced chemiluminescence system (Ab Frontier, Seoul, Korea). Full scans of western blots are in Supplementary Fig. 5.

Luciferase activity assay. Collected cells or isolated exosomes were washed in ice-cold PBS, and suspended in adjusted volumes of buffer provided with Reporter Lysis Buffer (Promega). A total volume of $20 \mu \mathrm{l}$ of samples was loaded in a black 96-well plate (SPL Life Sciences, Pocheon, Korea), and $100 \mu \mathrm{l}$ of Luciferase Assay Reagent (Promega)-reconstituted substrate was added to each reaction manually. Measurements were performed using a Berthold LB942 $\mathrm{TriStar}^{2}$ multidetection microplate reader (Berthold, Bad Wildbad, Germany). The integration time was set at $1 \mathrm{~s}$ for all measurements. To perform a quantitative luciferase assay, QuantiLum Recombinant Luciferase (Promega) was used to obtain a standard curve. On the basis of the standard curve, a fitting curve and the number of luciferase molecules were calculated.

Measurement of DNA-binding activity of NF-kB. Nuclear extracts $(5 \mu \mathrm{g})$ were assayed for the binding activity of p65/c-Rel (NF- $\mathrm{kB}$ ) using the TransAM NF- $\mathrm{kB}$ assay kit (ActiveMotif, Carlsbad, CA, USA) according to the manufacturer's manual ${ }^{32}$.

Culture of differentiated neurosphere-derived cells. The cerebral cortex tissues from E12.5 Rosa-EGFP reporter mice (The Jackson Laboratory, Bar Harbor, Maine, USA) were dissected from the brain ${ }^{33}$. Isolated tissues were incubated with Accutase (Innovative Cell Technologies, San Diego, CA, USA) for $5 \mathrm{~min}$ at $37^{\circ} \mathrm{C}$. Cells were grown on an ultralow attachment surface and maintained as neurospheres for 4 days in the presence of basic fibroblast growth factor $\left(20 \mathrm{ng} \mathrm{ml}^{-1}\right.$; R\&D systems, Minneapolis, MN, USA) and epidermal growth factor (20 $\mathrm{ng} \mathrm{ml}^{-1}$; Invitrogen) in DMEM/F12 (Gibco) media containing 1\% N2 (Gibco), $2 \%$ B27 (Gibco) supplements, and $1 \%$ penicillin-streptomycin (Gibco). For differentiation, neurospheres were dissociated and cultured on 18 - $\mathrm{mm}$ cover glass coated with poly-D-lysine $\left(100 \mathrm{mg} \mathrm{ml}^{-1}\right)$ and laminin $\left(5 \mathrm{mg} \mathrm{ml}^{-1}\right)$ with removal of epidermal growth factor/basic fibroblast growth factor. On the seventh day of differentiation, purified exosomes were treated to the cells and maintained the culture for an additional 3 days. For immunocytochemistry, cells were then fixed with $4 \%$ paraformaldehyde and immunostained with anti- $\beta$-Tubulin III (Tuj1) (diluted 1:1,000, Sigma-Aldrich, St. Louis, MO, USA, T2200), anti-GFP (diluted 1:1,000, Abcam, ab290) and counterstained with Hoechst 33342 (Invitrogen). Images were acquired on an EVOS FL cell imaging system (Invitrogen) with a EVOS AMEP 4624 (Plan Fluor $\times 20 / 0.45$ ) objective lens.

Animals. Animal care and handling were performed according to the guidelines of the Animal Care and Use Committee of the Korea Advanced Institute of Science and Technology (KAIST, Daejeon, Korea). Loxp-stop-loxp-eNpHR3.0-EYFP transgenic mice (The Jackson Laboratory) were generated by mating heterozygous transgenic and wild-type mice (Males, 22 weeks, C57BL/6J background) in a specific-pathogen-free environment. All mice were maintained on a $12 / 12$-h light/dark cycle (light cycle beginning at 6:00) at a temperature of $23^{\circ} \mathrm{C}$. Food and water were supplied ad libitum.

Stereotactic injection of EXPLORs in mouse brain. All mice were anaesthetized with avertin $\left(20 \mathrm{mg} \mathrm{ml}^{-1}\right.$ of tribromoethanol, $20 \mu \mathrm{g} \mathrm{g}^{-1}$ intraperitoneal, Sigma-Aldrich, Milwaukee, WI, USA) and placed in a stereotaxic apparatus (David Kopf Instruments, Tujunga, CA, USA). All injections were delivered at a rate of $0.3-0.8 \mu \mathrm{lmin}^{-1}$ via a $10-\mu \mathrm{l}$ Hamilton syringe (Hamilton Co., Reno, NV, USA) and an injection needle (33-gauge NanoFil Needle Assortment, blunt; WPI, Worcester, MA USA) using a syringe pump (KD Scientific, Holliston, MA, USA). For expression of eNpHR3.0-EYFP, control EXPLORs and Cre:EXPLORs were prepared. In total, $500 \mu \mathrm{g}$ of EXPLORs were injected into the ventrolateral part of the brain in each mouse $(-2.8 \mathrm{~mm}$ anteroposterior, -2.2 mediolateral and 3.8 dorsoventral). At 5 days after transfection, mice were killed for visualization.

Histology and immunohistochemistry of mouse brain. Mice were deeply anaesthetized with avertin $\left(20 \mathrm{mg} \mathrm{ml}^{-1}\right.$ of tribromoethanol, $20 \mu \mathrm{lg}^{-1}$ intraperitoneal, Sigma-Aldrich) and perfused first with heparin and then with $4 \%$ formaldehyde diluted in PBS. Brains were removed and post-fixed overnight at $4{ }^{\circ} \mathrm{C}$. Coronal sections (40- $\mu \mathrm{m}$ thick) were acquired using a vibratome (Leica VT1000S; Leica, Rockleigh, NJ, USA) and collected in PBS. Slices were mounted on glass slides with Vectashield mounting medium containing 4',6-diamidino-2phenylindole (DAPI; Vector Labs, Peterborough, UK). Fluorescent images (low magnification) were acquired with an Axio Imager 2 fluorescent microscope (Carl Zeiss). Confocal images were acquired with a LSM780 confocal microscope (Carl Zeiss) equipped with a Plan-Apochromat $\times 20 / 0.8$ M27 objective. Brain sections were incubated in PBS with 0.5\% Triton X-100 (Sigma-Aldrich) for $30 \mathrm{~min}$ at room temperature. After washing in PBS for $10 \mathrm{~min}$, sections were incubated in a blocking solution composed of $0.5 \%$ bovine serum albumin and $0.25 \%$ Triton-X 100 for $1 \mathrm{~h}$ at room temperature. For NeuN/GFAP immunostaining, sections were incubated with mouse anti-NeuN primary antibody (MAB377; Millipore) and chicken anti-GFAP primary antibody (AB5541; Millipore), diluted 1:1,000 in PBS containing $0.25 \%$ Triton X-100, for $15 \mathrm{~h}$ at room temperature. After washing twice in PBS for $5 \mathrm{~min}$ each, sections were incubated with fluorescein Alexa 647-conjugated anti-mouse IgG secondary antibody (Jackson ImmunoResearch, West Grove, PA, USA) and Alexa 555-conjugated anti-chicken IgG secondary antibody (Jackson ImmunoResearch), and diluted 1:300 in PBST for $1 \mathrm{~h}$ at room temperature. Sections were washed twice in PBS for 5 min each and mounted on glass slides with Vectashield mounting medium containing DAPI (Vector Labs).

Statistical analyses. Data are presented as means \pm s.e.m. The significance of the difference between two independent samples was determined using Student's $t$-test. Groups were compared using one-way analysis of variance, with Tukey's post hoc 
test applied to a significant main effect. All data were normally distributed and the variances were similar between the groups being statistically compared. Sample size was based on previous experience with experimental variability, and no statistical method was used to predetermine sample or animal sizes. No samples or animals were excluded from the analysis. All experiments were not randomized. The investigators were not blinded to allocation during experiments or outcome assessment.

Data availability. All data supporting the findings in this study are included in the article, either in the main figures or in the Supplementary Information files.

\section{References}

1. Mitragotri, S., Burke, P. A. \& Langer, R. Overcoming the challenges in administering biopharmaceuticals: formulation and delivery strategies. Nat. Rev. Drug Discov. 13, 655-672 (2014).

2. Becker-Hapak, M., McAllister, S. S. \& Dowdy, S. F. TAT-mediated protein transduction into mammalian cells. Methods 24, 247-256 (2001).

3. Kaczmarczyk, S. J., Sitaraman, K., Young, H. A., Hughes, S. H. \& Chatterjee, D. K. Protein delivery using engineered virus-like particles. Proc. Natl Acad. Sci. USA 108, 16998-17003 (2011).

4. Erazo-Oliveras, A. et al. Protein delivery into live cells by incubation with an endosomolytic agent. Nat. Methods 11, 861-867 (2014).

5. Wadia, J. S. \& Dowdy, S. F. Protein transduction technology. Curr. Opin. Biotechnol. 13, 52-56 (2002).

6. Eltoukhy, A. A. et al. Nucleic acid-mediated intracellular protein delivery by lipid-like nanoparticles. Biomaterials 35, 6454-6461 (2014).

7. Swaminathan, J. \& Ehrhardt, C. Liposomal delivery of proteins and peptides. Expert Opin. Drug Deliv. 9, 1489-1503 (2012).

8. Thery, C., Zitvogel, L. \& Amigorena, S. Exosomes: composition, biogenesis and function. Nat. Rev. Immunol. 2, 569-579 (2002).

9. Alvarez-Erviti, L. et al. Delivery of siRNA to the mouse brain by systemic injection of targeted exosomes. Nat. Biotechnol. 29, 341-345 (2011).

10. Ohno, S. et al. Systemically injected exosomes targeted to EGFR deliver antitumor microRNA to breast cancer cells. Mol. Ther. 21, 185-191 (2013).

11. Lai, C. P. et al. Visualization and tracking of tumour extracellular vesicle delivery and RNA translation using multiplexed reporters. Nat. Commun. 6, 7029 (2015).

12. Shalitin, D. et al. Regulation of Arabidopsis cryptochrome 2 by blue-lightdependent phosphorylation. Nature 417, 763-767 (2002).

13. Liu, H. et al. Photoexcited CRY2 interacts with CIB1 to regulate transcription and floral initiation in Arabidopsis. Science 322, 1535-1539 (2008).

14. Kennedy, M. J. et al. Rapid blue-light-mediated induction of protein interactions in living cells. Nat. Methods 7, 973-975 (2010).

15. Yazawa, M., Sadaghiani, A. M., Hsueh, B. \& Dolmetsch, R. E. Induction of protein-protein interactions in live cells using light. Nat. Biotechnol. 27, 941-U105 (2009).

16. Bugaj, L. J., Choksi, A. T., Mesuda, C. K., Kane, R. S. \& Schaffer, D. V. Optogenetic protein clustering and signaling activation in mammalian cells. Nat. Methods 10, 249-252 (2013).

17. Haney, M. J. et al. Specific transfection of inflamed brain by macrophages: a new therapeutic strategy for neurodegenerative diseases. PLOS ONE 8, e61852 (2013).

18. Shen, B., Wu, N., Yang, J. M. \& Gould, S. J. Protein targeting to exosomes/ microvesicles by plasma membrane anchors. J. Biol. Chem. 286, 14383-14395 (2011).

19. Yang, J. M. \& Gould, S. J. The cis-acting signals that target proteins to exosomes and microvesicles. Biochem. Soc. Trans. 41, 277-282 (2013).

20. Haney, M. J. et al. Exosomes as drug delivery vehicles for Parkinson's disease therapy. J. Control. Release 207, 18-30 (2015).

21. Hsu, Y. T., Wolter, K. G. \& Youle, R. J. Cytosol-to-membrane redistribution of Bax and Bcl-X(L) during apoptosis. Proc. Natl Acad. Sci. USA 94, 3668-3672 (1997).

22. Wolter, K. G. et al. Movement of Bax from the cytosol to mitochondria during apoptosis. J. Cell Biol. 139, 1281-1292 (1997).

23. Nechushtan, A., Smith, C. L., Hsu, Y. T. \& Youle, R. J. Conformation of the Bax C-terminus regulates subcellular location and cell death. EMBO J. 18, 2330-2341 (1999).

24. Gross, A., Jockel, J., Wei, M. C. \& Korsmeyer, S. J. Enforced dimerization of $\mathrm{BAX}$ results in its translocation, mitochondrial dysfunction and apoptosis. EMBO J. 17, 3878-3885 (1998).
25. Basak, S. et al. A fourth IkappaB protein within the NF-kappaB signaling module. Cell 128, 369-381 (2007).

26. Boehm, J. S. et al. Integrative genomic approaches identify IKBKE as a breast cancer oncogene. Cell 129, 1065-1079 (2007).

27. van den Berg, A. \& Dowdy, S. F. Protein transduction domain delivery of therapeutic macromolecules. Curr. Opin. Biotechnol. 22, 888-893 (2011).

28. Chen, T. S. et al. Enabling a robust scalable manufacturing process for therapeutic exosomes through oncogenic immortalization of human ESC-derived MSCs. J. Transl. Med. 9, 47 (2011).

29. Katsura, Y. et al. An optogenetic system for interrogating the temporal dynamics of Akt. Sci. Rep. 5, 14589 (2015).

30. Lee, S. et al. Reversible protein inactivation by optogenetic trapping in cells. Nat. Methods 11, 633-636 (2014).

31. Thery, C., Amigorena, S., Raposo, G. \& Clayton, A. Isolation and characterization of exosomes from cell culture supernatants and biological fluids. Curr. Protoc. Cell Biol. Ch. 3, Unit 3-22 (2006).

32. Yim, N. et al. Mutant ubiquitin UBB +1 induces mitochondrial fusion by destabilizing mitochondrial fission-specific proteins and confers resistance to oxidative stress-induced cell death in astrocytic cells. PloS ONE 9, e99937 (2014).

33. Shaker, M. R., Kim, J. Y., Kim, H. \& Sun, W. Identification and characterization of secondary neural tube-derived embryonic neural stem cells in vitro. Stem Cells Dev. 24, 1171-1181 (2015).

\section{Acknowledgements}

We thank Dr Hee-Seok Kweon (Korea Basic Science Institute, Daejeon, South Korea) for helping Immuno-EM analysis. This research was supported by Global Frontier Program through the National Research Foundation of Korea (NRF) funded by the Ministry of Science, ICT \& Future Planning (No. 2014M3A6A8063924) and Korea Health Technology R\&D Project through the Korea Health Industry Development Institute (KHIDI), funded by the Ministry of Health \& Welfare, Republic of Korea (grant number: HI14C0042).

\section{Author contributions}

N.Y. and C.C. designed the EXPLOR strategies and wrote the paper; C.C. and K.C. supervised the project; N.Y. built EXPLOR-related constructs and EXPLOR-producing system; N.Y., S.-W.R. and K.C. collected and analysed the data; N.Y., S.-W.R., K.C., K.R.L., S.L., H.C. and M.R.S. performed in vitro experiments; J.K. performed in vivo experiments; W.S., J.-H.P., D.K. and W.D.H. provided reagents, analysing tools and mice K.R.L., S.L. and H.C. gave technical support; J.H.P., D.K. and W.D.H. gave conceptual advice.

\section{Additional information}

Supplementary Information accompanies this paper at http://www.nature.com/ naturecommunications

Competing financial interests: C.C. is the scientific founder and Chief Executive Officer of Cellex Life Sciences Inc. S.-W.R. and K.R.L. are employees of Cellex Life Sciences Inc. A patent application has been submitted by KAIST based on these results. The remaining authors declare no competing financial interests.

Reprints and permission information is available online at http://npg.nature.com/ reprintsandpermissions/

How to cite this article: Yim, N. et al. Exosome engineering for efficient intracellular delivery of soluble proteins using optically reversible protein-protein interaction module Nat. Commun. 7:12277 doi: 10.1038/ncomms12277 (2016).

This work is licensed under a Creative Commons Attribution 4.0 International License. The images or other third party material in this article are included in the article's Creative Commons license, unless indicated otherwise in the credit line; if the material is not included under the Creative Commons license, users will need to obtain permission from the license holder to reproduce the material. To view a copy of this license, visit http://creativecommons.org/licenses/by/4.0/

(C) The Author(s) 2016 